\title{
Tumor Suppressors: Heroes and Villains?
}

\author{
Lindsay Hinck
}

Published online: 31 July 2011

(C) Springer Science+Business Media, LLC 2011

Great progress has been made in our understanding of tumor suppressor genes since landmark research by Knudson led to the "two-hit" hypothesis and the subsequent molecular identification of the retinoblastoma gene $(R b l)$ [1]. Knudson's 2-hit model, whereby germline mutation of one allele predisposes a tissue to tumor formation upon somatic mutation of the second allele, remains valid for many tumor suppressor genes. However, research in the intervening years has demonstrated that the model does not correctly describe the behavior of all tumor suppressors. For example, not all tumor suppressors must be fully inactivated in order to facilitate tumor initiation and progression. Consequently, there is an increasing appreciation of tumor suppressor genes being haploinsufficient. Moreover, it has become clear that tumor suppressors often function within signaling pathways, where they regulate complex cellular behaviors that impact the function of tissues, organs and, indeed, the entire organism. In these complex settings, so-called tumor suppressors can play a far more nuanced role by exerting either pro- or antitumorigenic effects depending on the biological context. In this issue, we tackle some of the complexities of tumor suppression by examining how biological processes (such as autophagy and innate immunity), environmental conditions (the microenvironment, parity, circadian cycles) intrinsic pathways (oncogene-induced senescence) and extrinsic cues (axon guidance molecules) influence the context-dependent action of these important genes. In some settings tumor suppressors play their designated role,

\section{Hinck $(\varangle)$}

Molecular, Cell \& Developmental Biology,

225 Sinsheimer Labs,

Santa Cruz, CA 95064, USA

e-mail: hinck@biology.ucsc.edu heroically keeping rogue cells in check, while in other contexts, they are villains that participate in tumor formation.

Autophagy, specifically macroautophagy in which cytoplasmic constituents are degraded and recycled through the lysosome, is the subject of the first review. This process is activated in response to multiple stresses occurring during tumor progression, including nutrient starvation, the unfolded protein response and hypoxia. Additionally, it is activated in response to cytotoxicity generated by cancer treatment. Consequently, at first glance, it appears that autophagy is villainous, functioning as a survival mechanism by facilitating nutrient recycling that helps cells endure the assaults associated with disease progression and treatment. However, other evidence paints a more complicated picture and supports a valiant role for autophagy in halting disease progression. At least one of the primary autophagy related genes, Becnl, appears to protect cells from genotoxic stress and maintain genome integrity under some conditions. Moreover, there is strong genetic evidence that Becn 1 is a tumor suppressor. The gene for Becn 1 maps to a tumor susceptibility locus that is mono-allelically deleted in a high percentage of human breast cancers, and its single copy loss in mice results in spontaneous tumor development. Together, these observations put Becnl in the class of haploinsufficient tumor suppressors whose function may be regulated by cellular context. In this issue, Jayantha Debnath discusses the multifaceted nature of autophagy in breast tumor progression, proposing a model in which the consequences of autophagy depend on tumor type, context and stage. The tumor suppressive functions of autophagy occur during tumor initiation, whereas its oncogenic role emerges at later stages of tumor progression as tumor cells cope with stresses from their microenvironment 
The theme of cellular context influencing the function of tumor suppressors continues in the second article in which Kees and Egeblad describe how breast tumors generate a detrimental microenvironment that inhibits innate immune cells from performing their natural protective functions. Properly activated, innate immune cells are heroes that effectively kill tumor cells. However, instead of eliminating their targets, innate immune cells are pushed towards the role of villain by the tumor and its surrounding stromal cells, which release anti-inflammatory cytokines and interfere with the innate immune response. The authors review the literature on cancer immunotherapy, including the pioneering work of the $19^{\text {th }}$ century surgeon, William Coley, who injected patients with bacteria or bacterial products, called Coley's Toxins, and successfully increased the survival of some cancer patients. These, and other more recent studies demonstrating limited but promising results in treating cancer with activated immune cells, lead Kees and Egeblad to propose a combined breast cancer treatment approach, involving innate immune system activation and concomitant inhibition of anti-inflammatory cytokines released by tumor cells. This treatment paradigm has proven promising in several contexts, including a mouse model of carcinoma, leading to renewed optimism in the field of cancer immunology.

The importance of the tumor microenvironment, specifically the extracellular matrix (ECM), is highlighted in the third article by Patricia Keely. Appreciation is growing for the numerous ways in which ECM contributes to tumor progression. By generating a strong fibrotic reaction, termed 'desmoplasia', mammary tumors become surrounded by deleterious stromal matrix components, such as fibrillar collagen types I, III and V. These become linearized and stiffer than the fibers surrounding normal tissue, and this, in turn, promotes tumor progression and metastasis by increasing growth factor signaling and fostering the migration of metastasizing tumor cells. Nevertheless, Keely tackles the subject from a fresh angle, positing a distinct role for ECM as a microenvironmental defender in normal breast, where it holds cells in the differentiated state. In this view, the ECM only becomes a tumor promoter during carcinogenesis when its composition changes dramatically due to degradation and inappropriate deposition of constituent proteins. Highlighted in the article are the roles of transmembrane proteins such as syndecan and integrin, as well as signaling pathways mediated by the small GTPase Rho and focal adhesion kinase.

In the next article, Russo and Russo tackle the importance of the systemic environment in breast cancer. Unlike some cancers that are influenced solely by their local microenvironment, breast cancer is under endocrine control and is affected profoundly by circulating hormones. These critical substances impact tumor progression in numerous ways, both as heroes and villains. For example, estrogen functions as a villain by supporting the growth of estrogen receptor-positive tumors, whereas, as discussed by Russo and Russo, human chorionic gonadotropin (hCG) or pregnancy acts heroically by inhibiting tumor development through the poorly understood process of parity-induced tumor suppression. The protective effect of early full-term pregnancy has been well established by epidemiological studies, but its molecular underpinnings are just now being revealed. Studies have shown that pregnancy generates extensive changes in the differentiation state of mammary epithelial cells. These changes include a permanent difference in the genomic signature that distinguishes parous from nulliparous breast tissue. The differentiation of breast epithelia generated by pregnancy is key to its protective effect, but this type of tumor suppression is temporally regulated. It is attainable only during a short developmental time period, requiring a complex interplay between the hormonal milieu and developmental state to achieve the genomic changes necessary to endow long-lasting protection against tumor development.

Circadian rhythm hormones also regulate tumorigenesis in a temporally-restricted manner. In the article by Hill and colleagues, the risks of "light-at-night" for breast cancer development and the tumor suppressive effects of melatonin are reviewed. Melatonin, a pineal neurohormone, is synthesized and secreted at night in response to darkness and is repressed during the day by light. Melatonin activates the MT1 and MT2 G-protein coupled receptors and, as described in the accompanying review, it inhibits the proliferation of breast cancer cells, in part, by repressing the transcriptional activity of estrogen receptor- $\alpha$. Other repressive effects of melatonin include its ability to inhibit the uptake and metabolism of potentially damaging polyunsaturated fatty acids, such as linoleic acid. Deregulation of signaling pathways downstream of melatonin appear to be the basis for the significantly elevated risk of breast cancer in women working night shifts. Hill and colleagues describe a unique experimental approach that is being used to investigate how "light-at-night" regulates the development and progression of breast cancer. These and other studies are shedding light on the importance of proteins whose expression is regulated by the master biological clock located in the suprachiasmatic nucleus of the hypothalamus. These proteins regulate the activity of organspecific peripheral clocks that are present in both normal and cancer cells, and regulate cellular processes involved in both tumor suppression and tumor promotion.

Next, Reddy and Li examine the tumor suppressive mechanism of oncogene-induced senescence (OIS), where- 
by cells respond to the villainous actions of oncogenes by undergoing cell cycle arrest through the activation of classic tumor suppressor pathways, such as those mediated by p16INK4A, RB1 and p53. OIS was originally discovered when cultured cells, expressing a mutant H-Ras ${ }^{\mathrm{V} 12}$, senesced prematurely, rather than progressing toward malignancy. As described in the accompanying review, in the intervening years the existence of OIS has been verified in vivo, and many interesting aspects of the process have been identified, including the mechanisms of its induction, its inactivation by tumor cells, and its associated secretory phenotype. Paradoxically, factors released as part of the senescence-associated secretory phenotype also exhibit growth-promoting effects that revert the hero into a villain by helping neighboring cells hyperproliferate. While OIS has been studied primarily in tumor models other than breast, recent research has shown that high levels of oncogenic $\mathrm{H}-\mathrm{Ras}^{\mathrm{V} 12}$ or overexpression of the mitotic kinase Aurora A in p53-null mice drives OIS in breast, suggesting this mechanism of tumor suppression also serves as a critical barrier to cancer in this organ.

The final article by Harburg and Hinck continues the theme of molecules acting as both friend and foe depending on the cellular context. Four families of guidance cues are discussed that were originally identified in the nervous system based on their ability to direct axonal migration. In this context, these secreted factors function as both attractants, capable of guiding axons toward targets, and repellents that generate exclusion zones. This functional duality has been conserved in solid tissues, such as breast, where guidance cues increase and decrease tumor cell adhe- sion, promote and suppress tumor cell metastasis, and stimulate and inhibit cell proliferation. These cues signal through receptors that feed into several common downstream pathways, including those mediated by PI3 kinase/Akt pathway and the Rho family of small GTPases. This regulation of central signaling pathways by axon guidance molecules, and the fact that several appear to be deregulated early in breast tumor progression, have made these molecules attractive candidates for diagnostic/prognostic markers, as well as promising therapeutic targets.

In conclusion, since Knudson's discovery of Rb1, the field of cancer biology has made great progress in understanding the complexity of tumor suppressors. As it turns out a half-century later, tumor suppressors do not always function as gene products that restrain tumor cell growth and motility. As described in this volume of papers, the circumstances in which tumor suppressors are deployed dictate their actions, and under some situations they promote, rather than inhibit, tumorigenesis. Although it is frustrating that researchers have been unable to simply define the actions of this class of genes, in the intervening years we have achieved a deeper understanding of their complex functions in tumors. These advances are leading to integrated, multi-pronged approaches for the treatment of breast cancer.

\section{Reference}

1. Knudson Jr AG. Mutation and cancer: statistical study of retinoblastoma. Proc Natl Acad Sci U S A. 1971;68(4):820-3. 